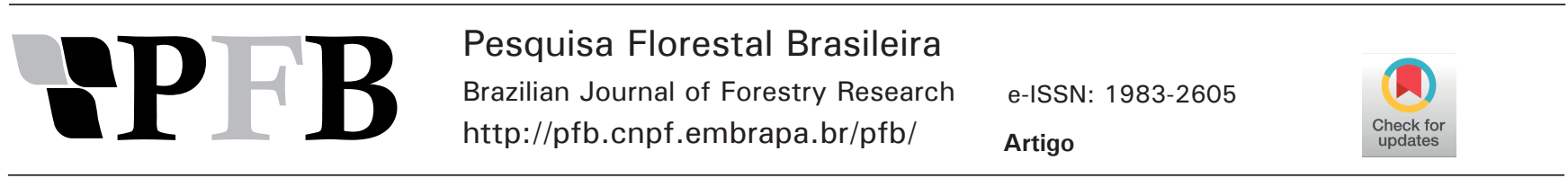

\title{
Equação de volume para espécies de Cerrado em Formosa, GO
}

\author{
Andreia Taborda dos Santos ${ }^{1}$ (D), Vitor Dressano Domene ${ }^{2}$ (D), Patrícia Póvoa de Mattos ${ }^{3 *}$ (iD), Evaldo Muñoz Braz ${ }^{3}$ (D), \\ Aline Canetti ${ }^{4}$ (i) , Nelson Yoshihiro Nakajima ${ }^{1}$ (i) , Nelson Carlos Rosot $^{1}$ (i) \\ ${ }^{1}$ Universidade Federal do Paraná, Avenida Prefeito Lothário Meissner, 3400, Jardim Botânico, CEP 80210-170, Curitiba, PR, Brasil \\ ${ }^{2}$ Mademape Indústria Madeireira Ltda, Av. Ricieri Bernanrdi, 635, Campo do Fundo, CEP 83430-000, Campina Grande do Sul, PR, Brasil \\ ${ }^{3}$ Embrapa Florestas, Estrada da Ribeira, km 111, C.P. 319, CEP 83411-000, Colombo, PR, Brasil \\ ${ }^{4}$ Consultora autônoma, Curitiba, PR, Brasil
}

"Autor correspondente:

patricia.mattos@embrapa.br

Termos para indexação:

Modelagem

Hohenadl-Krenn

Naslund modificado

Index terms:

Modelling

Hohenadl-Krenn

Naslund modified

Histórico do artigo:

Recebido em 17/12/2019

Aprovado em 05/10/2020

Publicado em 30/09/2021
Resumo - O presente trabalho teve como objetivo ajustar modelos para a estimativa de volume de espécies do Cerrado, em Formosa, GO. Foram selecionados de 12 a 15 indivíduos de nove espécies, representando a amplitude das classes diamétricas de cada espécie, na área de estudo. As árvores foram derrubadas e mensuradas por cubagem rigorosa e seus volumes foram calculados pelo método de Smalian. Foram testados sete modelos matemáticos para a estimativa do volume por espécie e total, sendo que o melhor modelo foi selecionado com base nos parâmetros estatísticos: coeficiente de determinação ajustado, erro padrão da estimativa e distribuição gráfica dos resíduos. Os modelos de Hohenadl-Krenn, de simples entrada, e Naslund modificado, de dupla entrada, apresentaram os melhores ajustes para a estimativa de volume de árvore individual das nove espécies estudadas e de todo conjunto de espécies.

\section{Wood volume model for Cerrado species from Formosa, Goias State, Brazil}

\begin{abstract}
This study aimed to develop models for estimating wood volume of individual trees for nine Cerrado species of Formosa, State of Goias, Brazil. Data were collected from 12 to 15 trees of nine species, representing the diametric classes amplitude of each species in the studying area. Trees were logged and measured and the volume was calculated by Smalian method. Seven mathematical models were tested to estimate the wood volume by species and total. The best model was selected through statistical parameters adjusted coefficient of determination, standard error of the estimate and graphical. distribution of residues. Hohenadl-Krenn (simple input) and Naslund modified (double input) presented the best results to estimate individual tree wood volume for nine studied species and also for all nine species together.
\end{abstract}

\section{Introdução}

O Cerrado originalmente cobria $23,9 \%$ do território brasileiro, em aproximadamente 200 milhões de ha (IBGE, 2004). Abrange 11 Estados e o Distrito Federal, sendo o segundo maior bioma brasileiro em extensão (IBF, 2020), e alimenta os três maiores aquíferos do mundo: Guarani, Bambuí e Urucuia (Bolson, 2018). Em um estudo realizado no período de 2011-2015 foi constatado que o desmatamento no Cerrado brasileiro foi superior ao da Floresta Amazônica (Bellantonio, et. al, 2017). O autor cita ainda que, em um período de quatro anos, mais de 567.000 ha do bioma Cerrado foram desmatados para dar lugar à produção de soja 
e à pecuária, e que a soja foi o principal motivo do desmatamento na região. Em um estudo realizado pelo Instituto Nacional de Pesquisas Espaciais (Inpe), no período de 2013-2015 e divulgado em 2017 pelo governo federal, verificou-se que o Cerrado perdeu $9.483 \mathrm{~km}^{2} \mathrm{de}$ vegetação, o que seria equivalente a mais de seis cidades do tamanho de São Paulo, superando em mais de 50\% a devastação na Amazônia, no ano de 2015.

Fatores edafoclimáticos e topográficos são os principais influenciadores da distribuição e da manutenção das diferentes fitofisionomias do bioma Cerrado (Eiten, 1972; Projeto Radambrasil, 1981). O bioma ocorre essencialmente sobre Latossolos e Neossolos Quartzarênicos profundos, bem drenados, distróficos, ácidos e álicos (Haridasan, 1982) e as principais características do solo que determinam as formações fitofisionômicas são profundidade efetiva, drenagem e fertilidade (Eiten, 1972; Haridasan, 1982).

Nos biomas brasileiros há espécies com as mais diferentes características silviculturais, ecológicas e tecnológicas, sendo poucas as informações de como essas plantas crescem, seja em áreas intactas, áreas exploradas ou em áreas sujeitas a regime de manejo (Scolforo et al., 1996). De acordo com Duboc (2008), na região do Cerrado concentra-se tanto a exploração de madeira nativa para a produção de carvão vegetal, quanto os maiores reflorestamentos destinados a suprir as necessidades energéticas do País.

A necessidade de desenvolvimento de ferramentas que propiciem informações relevantes ao planejamento do manejo sustentável surge com o aumento do interesse na utilização dos recursos oriundos de florestas naturais. Para entender e projetar a dinâmica da floresta, a modelagem provou ser uma ferramenta útil e importante e vem sendo desenvolvida e utilizada para prever a produtividade dos povoamentos florestais, estimar volumes, crescimento, entre outros (Higuchi \& Ramm, 1985; Huston et al., 1988; Vanclay, 1989; Borders et al., 1990; Mcmurtrie et al., 1990; Bossel, 1991; Prentice et al., 1993; Lima et al., 1996; Scolforo, 1996; Scolforo, 1997; Scolforo, 1998; Clark \& Clark, 2000; Silva et al., 2004; Eisfeld et al., 2005; Nogueira et al., 2006; Valério et al., 2007; Scolforo et al., 2008).

Muitas espécies arbóreas do Cerrado apresentam potencial para uso como fonte de energia, madeira sólida, produtos não madeiráveis ou mesmo para a recuperação de áreas degradadas. No entanto, simulações do estoque potencial ou do seu crescimento ou redução são difíceis, devido à carência de equações para a determinação do volume de madeira para muitas espécies arbóreas desses ambientes. A riqueza de espécies arbóreas desse bioma contrasta com a carência de informações sobre espécies arbóreas com potencial econômico, restringindo o seu aproveitamento (Durigan, 2003).

Conhecer o potencial volumétrico e a flora do bioma Cerrado é de extrema importância para a garantia de seu uso de forma sustentada, concomitantemente visando à conservação e à preservação do mesmo (Rufini et al., 2010). Preocupados com isso, pesquisadores buscaram determinar modelos para a estimativa de volume de espécies do Cerrado. Rezende et al. (2006) e ImañaEncinas et al. 2009 compararam modelos volumétricos para vegetação lenhosa do Cerrado, em Brasília. Scolforo et al. (2008) e Rufini et al. (2010) ajustaram modelos para estimar o volume total e do fuste, com casca e sem casca, para espécies do Cerrado, em Minas Gerais, no entanto, a tarefa é imensa, visto que existem mais de 1.130 espécies arbóreas madeireiras e não madeireiras, no bioma Cerrado, catalogadas até o momento (Pereira, 2017).

O presente trabalho teve como objetivo ajustar modelos de volume para árvore individual de nove espécies do Cerrado em Formosa, GO, em dois tipos de solos, visando ampliar o conhecimento sobre essas espécies e realizar estimativas precisas do volume de madeira das mesmas.

\section{Material e métodos}

$\mathrm{O}$ estudo foi realizado em uma área do Exército Brasileiro, no município de Formosa, GO, (15³2'14"S e 47²0'04'W), com altitude média de 917 m (IBGE, 2014).

A região está inserida no domínio do bioma Cerrado, onde a fitofisionomia dominante é o Cerrado stricto sensu, destacando-se ainda a presença do campo cerrado, mata seca e mata de galeria (Haridasan, 2000). No entanto, devido à proximidade com Brasília, DF, o índice de antropização regional é bem acentuado, com diversas alterações dos habitats da fauna e da flora. Em Formosa podem ser encontrado solos que, segundo a classificação brasileira de solos, são definidos como Latossolo Vermelho-Amarelo e o Latossolo Amarelo, não hidromórficos, caracterizados por apresentarem horizonte B latossólico (Santos et. al., 2018). Estes solos são muito profundos a profundos, acentuadamente drenados, muito permeáveis e porosos. Apresentam uma 
cobertura com concreções ferruginosas locais, sendo mais arenosos sobre os metarenitos alternados e ocorre em relevo plano à suave ondulado (Santos et al., 2006).

O clima da região é Cwa, tropical com estação seca no inverno (Köppen, 1936). A temperatura média anual é $21,7^{\circ} \mathrm{C}$, com mínima de $16,8^{\circ} \mathrm{C}$ e máxima de $27,7^{\circ} \mathrm{C}$. A precipitação pluviométrica média anual observada na região é $1.448 \mathrm{~mm}$, com o período de estiagem ocorrendo entre maio e setembro (Cardoso et al., 2014).

Foram selecionados, em média, 12 a 15 indivíduos adultos por espécie e por tipo de solo. As árvores, com fuste sem evidências de danos, foram cortadas e feita a cubagem rigorosa no campo, sendo medidos também a altura total com trena e o diâmetro a 1,30 m do solo com fita diamétrica. Da área de estudo foram selecionadas nove espécies, sendo elas: Anonna crassiflora Mart., Bowdichia virgilioides Kunth., Dimorphandra mollis Benth., Eugenia dysenterica (Mart.) DC., Kielmeyera coriacea Mart., Qualea grandiflora Mart., Q. parviflora Mart., Stryphnodendron adstringens Mart. e Tabebuia aurea (Silva Manso) Benth. \& Hook.

Foi efetuada a cubagem rigorosa de cada uma das árvores até 4,0 cm de diâmetro com casca, estabelecido como o limite mínimo de diâmetro comercial, semelhante ao utilizado por Sturion et al. (1988), Sturion \& Tomaselli (1990), Machado et al. (2008) e Santos et al. (2012). Com a medida dos diâmetros e o comprimento de cada segmento, calculou-se o volume total de madeira de cada árvore pelo método de Smalian.

Na tabela 1 são apresentados os valores do diâmetro da base médio, mínimo e máximo assim como os valores de altura média, mínima e máxima para cada espécie.

Para a estimativa dos volumes individuais com casca das árvores e do volume total das nove espécies selecionadas para o estudo, foram ajustados sete modelos matemáticos (Tabela 2), utilizando-se as árvores cubadas para o ajuste, sendo que destes modelos foram selecionados um de simples entrada e outro de dupla entrada.

Os dados foram analisados de forma conjunta e individualmente para cada uma das espécies, por meio de software estatístico. Foram selecionadas equações de simples e de dupla entrada, considerando aquelas que apresentaram os melhores parâmetros estatísticos do ajuste de regressão (coeficiente de determinação ajustado $\mathrm{R}^{2}$ aj. e erro padrão da estimativa Syx \%, além dos parâmetros $\beta_{0}, \beta_{1}, \beta_{2}, \beta_{3}$ e $\beta_{4}$ ) e adequação visual aos dados reais por meio da análise dos resíduos, que consiste em avaliar o comportamento dos dados estimados pelo modelo diante dos dados reais, verificando as divergências entre eles.

Tabela 1. Valores médios, mínimos e máximos do diâmetro da base $(\mathrm{Db})$ e altura das árvores $(\mathrm{H})$ para cada espécie estudada em vegetação de Cerrado em Formosa, GO.

Table 1. Average, minimum and maximum values of the diameter at the ground level $(\mathrm{Db})$ and height $(\mathrm{H})$ for each species studied in savanna vegetation, in Formosa, Goias State.

\begin{tabular}{|c|c|c|c|c|c|c|}
\hline Espécie & $\begin{array}{c}\text { Db } \\
\text { médio } \\
\text { (cm) }\end{array}$ & $\begin{array}{c}\text { Db } \\
\text { minimo } \\
(\mathrm{cm})\end{array}$ & $\begin{array}{c}\text { Db } \\
\text { máximo } \\
(\mathrm{cm})\end{array}$ & $\begin{array}{c}\text { H } \\
\text { média } \\
(\mathbf{m})\end{array}$ & $\begin{array}{c}\text { H } \\
\text { mínima } \\
\text { (m) }\end{array}$ & $\begin{array}{c}\text { H } \\
\text { máxima } \\
(\mathrm{m})\end{array}$ \\
\hline $\begin{array}{l}\text { Anonna } \\
\text { crassiflora }\end{array}$ & 16,75 & 11,46 & 26,10 & 5,15 & 3,20 & 6,30 \\
\hline $\begin{array}{l}\text { Bowdichia } \\
\text { virgilioides }\end{array}$ & 18,06 & 10,50 & 37,59 & 7,68 & 4,20 & 14,10 \\
\hline $\begin{array}{l}\text { Dimorphandra } \\
\text { mollis }\end{array}$ & 8,88 & 6,21 & 13,05 & 4,02 & 3,00 & 5,60 \\
\hline $\begin{array}{l}\text { Eugenia } \\
\text { dysenterica }\end{array}$ & 18,50 & 9,71 & 42,97 & 5,90 & 3,70 & 7,30 \\
\hline $\begin{array}{l}\text { Kielmeyera } \\
\text { coriacea }\end{array}$ & 10,85 & 7,00 & 14,64 & 3,73 & 2,53 & 5,80 \\
\hline $\begin{array}{l}\text { Qualea } \\
\text { grandiflora }\end{array}$ & 16,51 & 9,23 & 21,33 & 5,34 & 3,10 & 7,30 \\
\hline $\begin{array}{l}\text { Qualea } \\
\text { parviflora }\end{array}$ & 16,75 & 7,96 & 25,78 & 6,24 & 3,74 & 7,90 \\
\hline $\begin{array}{l}\text { Stryphnodendron } \\
\text { adstringens }\end{array}$ & 11,42 & 6,88 & 15,28 & 4,13 & 2,50 & 5,60 \\
\hline Tabebuia aurea & 11,50 & 7,96 & 14,96 & 3,60 & 2,60 & 4,80 \\
\hline
\end{tabular}

Tabela 2. Modelos testados para a estimativa de volume individual de árvores de Cerrado em Formosa, GO.

Table 2. Tested models for estimating the individual volume of savanna trees in Formosa, Goias State.

\begin{tabular}{cc}
\hline Autores & Modelos volumétricos \\
\hline Bernac & $\ln v o l=\beta_{0}+\beta_{1} \ln d+\beta_{2} \frac{1}{d}$ \\
Hohenadl-Krenn & $v o l=\beta_{0}+\beta_{1} d+\beta_{2} d^{2}$ \\
Husch & $\ln v o l=\beta_{0}+\beta_{1} \ln d$ \\
Kopezki-Gehardt & $v o l=\beta_{0}+\beta_{1} d^{2}$ \\
Naslund modificado & $v o l=\beta_{0}+\beta_{1} d^{2}+\beta_{2} d^{2} h+\beta_{3} d h^{2}+\beta_{4} h^{2}$ \\
Schumacher-Hall & $\ln v o l=\beta_{0}+\beta_{1} \ln d+\beta_{2} \ln h$ \\
Spurr & $\ln v o l=\beta_{0}+\beta_{1} \ln d^{2} h$
\end{tabular}

Sendo: vol $=$ volume de madeira estimado para árvore individual; DAP $=$ diâmetro a $1,30 \mathrm{~m}$ do solo; $\mathrm{h}=$ altura total, $\beta_{n}=$ parâmetros da equação, $\ln =$ logaritmo neperiano. Fonte: Finger (1992). 


\section{Resultados}

Foram derrubadas e cubadas de 12 a 15 árvores para cada espécie, com DAP entre $6,21 \mathrm{~cm}$ e $42,97 \mathrm{~cm}$ e altura entre $2,50 \mathrm{~m}$ e $14,10 \mathrm{~m}$ (Tabela 1). Foram ajustadas equações de volume com casca de árvore individual de cada espécie, independentemente do tipo de solo em que se encontravam (Latossolo Amarelo ou Latossolo Vermelho). A seguir, foram ajustadas equações de volume por tipo de solo. Os parâmetros estatísticos das equações de volume de madeira selecionados para cada espécie, independentemente do tipo de solo, estão presentes na Tabela 3.

Para a maioria das espécies estudadas, constatou-se que o modelo de simples entrada que mais se aproximou do valor real foi o de Hohenald-Krenn. Entre os modelos de dupla entrada o que estimou melhor o volume das espécies foi o de Naslund modificado.

Os parâmetros estatísticos dos modelos de volume testados para as nove espécies em conjunto estão apresentados na Tabela 4. Os modelos que apresentaram os melhores resultados para os parâmetros estatísticos foram os de Hohenadl-Krenn e de Kopezki-Gehrardt para simples entrada e Naslund modificado para dupla entrada. Entretanto, dentre os modelos de simples entrada, o modelo de Hohenadl-Krenn foi selecionado por apresentar uma melhor distribuição dos resíduos (Figura 1). O volume total estimado para todas as espécies usando o modelo de simples entrada foi $99,97 \%$ $\left(13,25 \mathrm{~m}^{3}\right)$ do volume real e para modelo de dupla entrada o volume estimado foi $99,34 \%\left(13,17 \mathrm{~m}^{3}\right)$ do volume real, que era de $13,26 \mathrm{~m}^{3}$.

Tabela 3. Parâmetros estatísticos dos modelos de simples e de dupla entrada de volume de madeira para árvore individual, selecionados para cada uma das espécies estudadas, em vegetação de Cerrado, em Formosa, GO.

Table 3. Statistical parameters of the individual tree volume models tested for each of the species studied in savanna vegetation, in Formosa, Goias State.

\begin{tabular}{|c|c|c|c|c|c|c|c|}
\hline Modelo & $\mathbf{R}^{2} \mathbf{a j}$. & Syx\% & $\boldsymbol{\beta}_{0}$ & $\beta_{1}$ & $\beta_{2}$ & $\beta_{3}$ & $\beta_{4}$ \\
\hline \multicolumn{8}{|c|}{ Anonna crassiflora } \\
\hline Hohenald-Krenn & 0,86 & 36,0 & $-1,0073$ & 0,1473 & $-0,0042$ & - & - \\
\hline Naslund modificado & 0,90 & 41,0 & 0,4597 & $-0,0029$ & $-0,0008$ & $-0,0075$ & $-0,0660$ \\
\hline \multicolumn{8}{|c|}{ Bowdichia virgilioides } \\
\hline Hohenald-Krenn & 0,99 & 10,0 & 0,3130 & $-0,0452$ & 0,0020 & - & - \\
\hline Naslund modificado & 0,99 & 5,0 & $-0,0345$ & $-0,0003$ & $8,4 \cdot 10^{-5}$ & $-7,2.10^{-5}$ & 0,0023 \\
\hline \multicolumn{8}{|c|}{ Dimorphandra mollis } \\
\hline Hohenald-Krenn & 0,96 & 9,7 & 0,1448 & $-0,0130$ & 0,0003 & - & - \\
\hline Naslund modificado & 0,98 & 8,7 & 0,0113 & $-0,0002$ & 0,0001 & $-0,0005$ & 0,0058 \\
\hline \multicolumn{8}{|c|}{ Eugenia dysenterica } \\
\hline Hohenald-Krenn & 0,96 & 9,7 & 0,1448 & $-0,0130$ & 0,0003 & - & - \\
\hline Naslund modificado & 0,98 & 8,7 & 0,0113 & $-0,0002$ & 0,0001 & $-0,0005$ & 0,0058 \\
\hline \multicolumn{8}{|c|}{ Kielmeyera coriacea } \\
\hline Hohenald-Krenn & 0,99 & 2,0 & $-0,0198$ & 0,0023 & 0,0026 & - & - \\
\hline Naslund modificado & 0,99 & 5,0 & $-0,0151$ & 0,0003 & $3,34 \cdot 10^{-5}$ & $-0,0001$ & 0,0014 \\
\hline \multicolumn{8}{|c|}{ Qualea grandiflora } \\
\hline Kopezki-Gehrardt & 0,99 & 4,0 & $-0,0251$ & 0,0006 & - & - & - \\
\hline Naslund modificado & 0,99 & 3,0 & $-0,0052$ & $4,6.10^{-5}$ & $9,7 \cdot 10^{-5}$ & $-5,9 \cdot 10^{-5}$ & 0,0002 \\
\hline \multicolumn{8}{|c|}{ Qualea parviflora } \\
\hline Hohenald-Krenn & 0,99 & 5,0 & 0,0450 & $-0,0113$ & 0,0011 & - & - \\
\hline Naslund Modificado & 0,99 & 5,0 & 0,0178 & $-0,0001$ & 0,0001 & $-6,8 \cdot 10^{-5}$ & $-0,0003$ \\
\hline \multicolumn{8}{|c|}{ Stryphnodendron adstringens } \\
\hline Kopezki-Gehrardt & 0,98 & 5,0 & $-0,0092$ & 0,0004 & - & - & - \\
\hline Naslund modificado & 0,99 & 5,0 & $-0,0071$ & 0,0009 & $-0,0003$ & 0,0006 & $-0,0026$ \\
\hline \multicolumn{8}{|c|}{ Tabebuia aurea } \\
\hline Hohenald-Krenn & 0,81 & 19,0 & 0,0790 & $-0,0120$ & 0,0006 & - & - \\
\hline Naslund modificado & 0,98 & 9,0 & $-0,0051$ & $-0,0012$ & 0,0012 & $-0,0032$ & 0,0167 \\
\hline
\end{tabular}

Sendo: $\mathrm{R}^{2}$ aj. = coeficiente de determinação ajustado; $\mathrm{Syx} \%=$ erro padrão da estimativa; $\beta n=$ parâmetros da equação. 
Tabela 4. Parâmetros estatísticos dos modelos de volume testados para o conjunto de dados das nove espécies estudadas em vegetação de Cerrado em Formosa, GO.

Table 4. Statistical parameters of the tested volume models for the data set of the nine species studied in Cerrado vegetation, in Formosa, GO State.

\begin{tabular}{cccccccc}
\hline Modelo & $\mathbf{R}^{2} \mathbf{a j}$ & $\mathbf{S y x} \mathbf{0}$ & $\boldsymbol{\beta}_{\mathbf{0}}$ & $\boldsymbol{\beta}_{\mathbf{1}}$ & $\boldsymbol{\beta}_{\mathbf{2}}$ & $\boldsymbol{\beta}_{\mathbf{3}}$ & $\boldsymbol{\beta}_{\mathbf{4}}$ \\
\hline Brenac & 0,90 & 45,4 & $-9,1717$ & 2,5224 & $-0,5594$ & - & - \\
Hohenadl-Krenn & 0,82 & 7,5 & 0,0127 & $-0,0049$ & 0,0008 & - & - \\
Husch & 0,90 & 45,0 & $-9,3423$ & 2,5708 & - & - & - \\
Kopezki-Gehrardt & 0,82 & 7,5 & $-0,0227$ & 0,0006 & - & $-0,0001$ & - \\
Naslund modificado & 0,85 & 7,1 & $-0,0114$ & $6,4.10^{-5}$ & $8,4.10^{-5}$ & - & - \\
Schumacher-Hall & 0,93 & 39,1 & $-9,4028$ & 1,9418 & 1,0167 & - & - \\
Spurr & 0,93 & 38,9 & $-9,41254$ & 0,9836 & - & -
\end{tabular}

Sendo: $\mathrm{R}^{2}$ aj. = coeficiente de determinação ajustado; Syx\% = erro padrão da estimativa; $\beta n$ = parâmetros da equação.
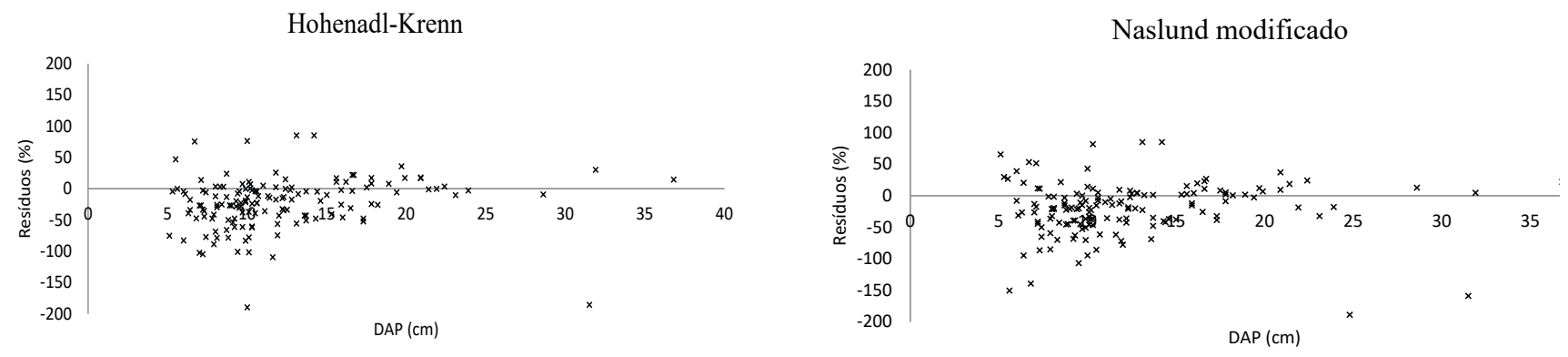

Figura 1. Distribuição dos resíduos dos melhores modelos testados para a estimativa do volume de árvore individual de Cerrado em Formosa, GO.

Figure 1. Residues distribution of the best models used for estimating the wood volume of individual trees from savanna in Formosa, Goias State.

\section{Discussão}

$O$ erro padrão da estimativa em porcentagem encontrado $($ Syx \%), apesar de elevado, pode ser considerado aceitável, por se tratar de área de vegetação natural (Tabela 3). Scolforo et al. (2008) e Morais et al. (2014) encontraram valores semelhantes de Syx\% em florestas naturais em Minas Gerais. De acordo com Rezende et. al. (2006), as árvores do Cerrado apresentam troncos grossos e tortuosos, sendo esses atributos típicos do Cerrado stricto sensu, onde o mesmo é caracterizado por vegetação arbórea esparsa, ocorrendo diversidade entre espécies e dentro da mesma espécie, o que leva a um elevado valor do erro padrão da estimativa (Syx\%). A fitofisionomia dominante da área de estudo é o Cerrado stricto sensu. Entretanto, a região está muito próxima de Brasília, DF, onde se observa a presença do campo cerrado, mata seca e mata de galeria, além da antropização elevada da região, o que contribui ainda mais para a variabilidade das amostras.

As estimativas de volume de madeira com casca por meio dos modelos de simples entrada foram semelhantes às estimativas de volume obtidas com os modelos de dupla entrada, para todas as espécies estudadas. Os modelos de simples entrada são recomendados quando apresentam bons ajustes, por dependerem apenas da variável diâmetro que é de fácil mensuração no campo e por não haver a necessidade de variável hipsométrica, o que torna as medições muito mais rápidas e baratas nos inventários florestais (Machado et al., 2008).

Em Cerrado stricto sensu de Minas Gerais, na região da bacia do Rio São Francisco, com 495 árvores cubadas em oito classe de diâmetros diferentes, Rufini 
et al. (2010) relataram como os melhores modelos para estimar volume real de madeira os de Schumacher-Hall e Spurr, em suas formas logarítmicas. Em outra área de Cerrado stricto sensu em Minas Gerais, Scolforo et al. (2008) também recomendaram o modelo de Schumacher-Hall em sua forma logarítmica para estimar o volume de madeira.

Em função do pequeno número de árvores amostradas por espécie, os resultados obtidos neste trabalho não permitem generalizações definitivas, entretanto os mesmos são consistentes para seleção da melhor equação. Desta maneira, para a seleção da equação mais adequada, é importante se atentar a algumas particularidades como o sítio, a quantidade e a qualidade dos indivíduos amostrados bem como o objetivo pelo qual está se fazendo a estimativa do modelo.

As plantas que crescem em Latossolos Amarelos, apesar de terem maior umidade disponível, apresentam limitação do crescimento em virtude da saturação dos poros nos períodos de maior precipitação pluvial ao longo do ano, o que acarreta dificuldade de desenvolvimento (Lopes, 1984). Por outro lado, nos Latossolos Vermelhos a fertilidade é maior, apesar da maior restrição de água ao longo do ano. Kozlowski et al. (1991) ressaltam que o crescimento e o rendimento de todos os tipos de plantas variam amplamente devido às diferenças na qualidade do local ou na capacidade produtiva do solo em que estão crescendo. Características gerais da complexa estrutura das comunidades vegetais e a riqueza de espécies estão potencialmente relacionadas às condições e padrões associados à umidade do solo e outros fatores do meio (Goodland \& Pollard, 1973; Hall \& Swaine, 1976). Haridasan, (2000) enfatiza que algumas espécies ocorrem somente em solos ácidos, outras são restritas aos solos calcários e outras indiferentes quanto à fertilidade do solo.

Informações precisas e confiáveis sobre as caracteristicas das espécies florestais nativas são de extrema importância para a definição de políticas e estratégias públicas a serem aplicadas como instrumentos de controle e manejo de remanescentes florestais (Scolforo et al., 2015). Assim, ferramentas dendrométricas como a modelagem devem ser conduzidas em ambientes distintos de vegetação do Cerrado, visando garantir informações consistentes para subsidiar a produção em inventários, determinar boas práticas de manejo, e garantir decisões corretas e estratégicas em planos florestais.
Em função da carência de informações sobre as espécies do Cerrado, é interessante que novos estudos sejam realizados para dar suporte ao uso sustentável dos recursos naturais que esse bioma oferece, e evitar que ele seja ainda mais degradado.

\section{Conclusões}

As equações desenvolvidas para as nove espécies apresentaram parâmetros estatísticos satisfatórios, com estimativas próximas ao volume real de madeira. As equações podem ser utilizadas na região de influência para a avaliação volumétrica de levantamentos florestais pois, mesmo havendo dois tipos de solos diferentes na área de estudo, não houve diferença do modelo escolhido para cada espécie.

A equação de Hohenadl-Krenn mostrou-se satisfatória para representar o volume real observado na área de estudo e também interessante por utilizar apenas o diâmetro, que é uma variável de fácil e rápida mensuração, reduzindo os custos dos levantamentos.

Devido à variabilidade natural dos locais amostrados, recomenda-se que novos estudos com amostragem maior sejam conduzidos, com enfoque especial em diferentes tipos de solo, o que permitirá investigar se os modelos seriam diferentes daqueles selecionados neste trabalho.

\section{Agradecimentos}

À Confederação da Agricultura e Pecuária do Brasil e à Empresa Brasileira de Pesquisa Agropecuária, executores do projeto Biomas onde está inserida a atividade de pesquisa que resultou neste manuscrito, e ao Senar, Sebrae, Monsanto e John Deere, patrocinadores do projeto Biomas.

\section{Referências}

Bellantonio, M. et al. O maior mistério da cadeia de produção de carne: os segredos por trás do Burger King e da produção mundial de carne. [S.1]: Rainforest Foundation Norway, Mighty Earth, [2017]. Disponível em: http://www.mightyearth.org/mysterymeatportuguese. Acesso em: 22 maio 2020.

Bolson, S. H. O Cerrado nas metas brasileiras do acordo de Paris: a omissão do estado brasileiro com o desmatamento na Cumeeira da América do Sul. Revista de Direito Ambiental e Sócio Ambientalismo, v. 4, n.1, p.112-131, 2018. http://dx.doi. org/10.26668/IndexLawJournals/2525-9628/2018.v4i1.3996. 
Borders, B. E. et. al. Yield prediction and growth projection for site-prepared loblolly pine plantations in the Carolinas, Georgia, Florida, and Alabama. Athens: School of Forest Resources, University of Georgia, 1990. (Research paper, 1990- 2).

Bossel, H. et. al. Simulation of forest stand dynamics, using realstructure process models. Forest Ecology and Management, v. 42, n. 1-2, p. 3-21, 1991. https://doi.org/10.1016/0378-1127(91)90061-Y.

Cardoso, M. R. D. et. al. Classificação climática de Köppen-Geiger para o estado de Goiás e o Distrito Federal. Acta Geográfica, v. 8, n. 16, p. 40-55. 2014. http://dx.doi.org/10.5654/acta.v8i16.1384.

Clark, D. B. \& Clark, D. A. Landscape-scale variation in forest structure and biomass in a tropical rain forest. Forest ecology and management, v. 137, n. 1-3, p. 185-198, 2000. http://dx.doi. org/10.1016 S0378-1127 (99) 00327-8.

Duboc, E. O Cerrado e o Setor Florestal. Planaltina, DF: Embrapa Cerrados, 2008.

Durigan, G. Bases e diretrizes para restauração da vegetação de cerrado. In: Kageyama, D. Y. et al. (Org.). Restauração ecológica de ecossistemas naturais. Botucatu: Fundação de Estudos e Pesquisas Agrícolas e Florestais, p. 185-204, 2003.

Eisfeld, R. L. et. al. Modelagem do crescimento e da produção de Pinus taeda L. por meio de função probabilística. Floresta, v. 35, n. 2, p. 317-328, 2005. http://dx.doi.org/10.5380/rf.v35i2.4619.

Eiten, G. The cerrado vegetation of Brazil. The Botanical Review, v. 38 , n. 2, p. 201-341, 1972.

Finger, C. A. G. Fundamentos de biometria florestal. UFSM, Santa Maria, 1992, 269 p.

Goodland, R. \& Pollard, R. The Brazilian cerrado vegetation: a fertility gradient. The Journal of Ecology, p. 219-224, 1973. https:// doi.org/10.2307/2258929.

Hall, J. B. \& Swaine, M. Classification and ecology of closed-canopy forest in Ghana. The Journal of Ecology, p. 913-951, 1976. https:// doi.org/10.2307/2258816.

Haridasan, M. Aluminium accumulation by some cerrado native species of central Brazil. Plant and soil. v. 65, n. 2, p. 265-273, 1982. https://doi.org/10.1007/BF02374657.

Haridasan, M. Nutrição mineral de plantas nativas do Cerrado. Revista Brasileira de Fisiologia Vegetal, Campinas, v. 12, n. 1, p. 54-64, 2000.

Higuchi, N. \& Ramm, W. Developing bole wood volume equations for a group of tree species of central Amazon (Brazil). Common wealth Forestry. v. 64, n. 1, p. 33-41, 1985.

Huston, M. et al. New computer models unify ecological theory. BioScience. v. 38, n. 10, p. 682-691, 1988. https://doi.org/10.2307 /1310870.

IBGE. Mapas de vegetação do Brasil. Rio de Janeiro, 2004.

IBGE. Cidades. Rio de Janeiro, 2014.

Imaña Encinas, J. et. al. Equações de volume de madeira para o cerrado de Planaltina de Goiás. Floresta. v. 39, n. 1, p. 107-116, 2009. http://dx.doi.org/10.5380/rf.v39i1.13731.
Instituto Brasileiro de Florestas. Bioma Cerrado. https://www. ibflorestas.org.br/bioma-cerrado. Acesso em 13/11/2020.

Köppen, W. Das geographische system der klimatologie. Berlin, 1936. $44 \mathrm{p}$.

Kozlowski, T. T. et. al. The physiological ecology of woody plants. San Diego: Academic Press, 1991. 657 p.

Lima, P. C. F. et. al. Equações para estimativa de biomassa de espécies de Prosopis no semi-árido brasileiro. Boletim de Pesquisa Florestal, n. 32/33, p. 67-79, 1996.

Lopes, A. S. Solos sob cerrado: características, propriedades e manejo. 162 p. 1984.

Machado, S. A. et. al. Modelagem volumétrica para bracatinga (Mimosa scabrella) em povoamentos da Região Metropolitana de Curitiba. Pesquisa Florestal Brasileira. n. 56, p. 17-29, 2008.

McMurtrie, R. E. et. al. Modelling the yield of Pinus radiata on a site limited by water and nitrogen. Forest Ecology and Management, v. 30, p.381-413, 1990. https://doi.org/10.1016/0378-1127(90)90150-A.

Nogueira, E. M. et. al. Volume and biomass of trees in central Amazonia: influence of irregularly shaped and hollow trunks. Forest Ecology and Management. v. 227, n. 1, p. 14-21, 2006. https://doi. org/10.1016/j.foreco.2006.02.004.

Pereira, B. A.S. Arvores do Bioma Cerrado, 2017. Disponível em http://www.arvoresdobiomacerrado.com.br/site/lista-de-especies/. Acesso em: 29 nov. 2019.

Prentice, I. C. et. al. A Simulation Model for the Transient Effects of Climate Change on Forest Landscapes. Ecological Modelling. v. 65, p. 51-70, 1993.

PROJETO RADAMBRASIL. Folha SD.22 Goiás: geologia, geomorfologia, pedologia, vegetação, uso potencial da terra. Rio de Janeiro: Ministério das Minas e Energia, Secretaria-Geral, 1981.6636 p. (Levantamento de recursos naturais, 25)

Reatto, A. et. al. Solos do bioma Cerrado: aspectos pedológicos. Cerrado: ambiente e flora, p. 47-86, 1998.

Rezende, A. V. et al. Comparação de modelos matemáticos para estimativa do volume, biomassa e estoque de carbono da vegetação lenhosa de um cerrado sensu stricto em Brasília, DF. Scientia Forestalis, v. 71, p. 65-76, 2006.

Rufini, A. L. et. al. Equações volumétricas para o cerrado sensu stricto, em Minas Gerais. Cerne. v. 16, n. 1, p. 1-11, 2010. https:// doi.org/10.1590/S0104-77602010000100001.

Santos, A. T. et al. Equação de volume e relação hipsométrica para plantio de Ocotea porosa. Pesquisa Florestal Brasileira, v. 32, n. 69, p. 13, 2012. https://doi.org/10.4336/2012.pfb.32.69.13.

Santos, H. G. dos et al. (Ed.). Sistema Brasileiro de Classificação de Solos. 2. ed. Rio de Janeiro: Embrapa Solos, 2018. 306 p.

Scolforo, J. R. S. et. al. Modelo de produção para floresta nativa como base para manejo sustentado. Cerne. v. 2, n. 1, 1996.

Scolforo, J. R. S. Técnica de regressão aplicada para estimar: volume, biomassa, relação hipsométrica e múltiplos produtos de madeira. Lavras: ESAL: FAEPE. 292 p., 1997. 
Scolforo, J. R. S. Modelagem do crescimento e da produção de florestas plantadas e nativas. Lavras: UFLA/FAEPE, 1998, 441 p.

Scolforo, J. R. et. al. Equações para estimar o volume de madeira das fisionomias, em Minas Gerais. In: Scolforo, J. R. et. al. Inventário florestal de Minas Gerais: equações de volume, peso de matéria seca e carbono para diferentes fisionomias da flora nativa. Lavras: UFLA, p.67-101, 2008.

Scolforo, H. F. et. al. Spatial distribution of aboveground carbon stock of the arboreal vegetation in Brazilian biomes of Savanna, Atlantic Forest and SemiArid Woodland. PLoS ONE 10, v. 6, p. 1-20, 2015. https://doi.org/10.1371/journal.pone.0128781.

Silva, H. D. et. al. Alocação de biomassa e ajuste de equações para estimativa de biomassa em compartimentos aéreos de Eucalyptus benthamii. Boletim de Pesquisa Florestal, n. 49, p. 83-95, 2004.
Sturion, J. et. al. Qualidade da madeira de Eucalyptus vimanalis para fins energéticos em função do espaçamento e idade de corte. Boletim de Pesquisa Florestal, n. 16, p. 55-59, 1988.

Sturion, J. A. \& Tomaselli, I. Influência do tempo de estocagem de lenha de bracatinga na produção de energia. Boletim de Pesquisa Florestal, n. 21, p. 37-47, 1990.

Valério, A. F. et. al. Ajuste de modelos matemáticos para estimativa de biomassa nos distintos componentes de uva do japão (Hovenia dulcis Thunb.). Ambiência, v. 3, n. 3, p. 325-336, 2007.

Vanclay, J. K. A growth model for north Queensland rainforests. Forest Ecology and Management. v. 27, p. 245-271, 1989. https:// doi.org/10.1016/0378-1127(89)90110-2. 